\title{
Origin of Predominance of Cementite among Iron Carbides in Steel at Elevated Temperature
}

\author{
C. M. Fang, ${ }^{1,2, *}$ M. H. F. Sluiter, ${ }^{3}$ M. A. van Huis, ${ }^{1,4}$ C. K. Ande, ${ }^{2,3}$ and H. W. Zandbergen ${ }^{1}$ \\ ${ }^{1}$ Kavli Institute of Nanoscience, Delft University of Technology, Lorentzweg 1, 2628 CJ Delft, The Netherlands \\ ${ }^{2}$ Materials Innovation Institute (M2i), Mekelweg 2, 2628 CD Delft, The Netherlands \\ ${ }^{3}$ Department of Materials Science and Engineering, Delft University of Technology, Mekelweg 2, 2628 CD Delft, The Netherlands. \\ ${ }^{4}$ EMAT, University of Antwerp, Groenenborgerlaan 171, 2020 Antwerp, Belgium
}

(Received 2 February 2010; published 30 July 2010)

\begin{abstract}
A long-standing challenge in physics is to understand why cementite is the predominant carbide in steel. Here we show that the prevalent formation of cementite can be explained only by considering its stability at elevated temperature. A systematic highly accurate quantum mechanical study was conducted on the stability of binary iron carbides. The calculations show that all the iron carbides are unstable relative to the elemental solids, $\alpha$-Fe and graphite. Apart from a cubic $\mathrm{Fe}_{23} \mathrm{C}_{6}$ phase, the energetically most favorable carbides exhibit hexagonal close-packed $\mathrm{Fe}$ sublattices. Finite-temperature analysis showed that contributions from lattice vibration and anomalous Curie-Weis magnetic ordering, rather than from the conventional lattice mismatch with the matrix, are the origin of the predominance of cementite during steel fabrication processes.
\end{abstract}

DOI: 10.1103/PhysRevLett.105.055503

Despite the fact that steel is one of the most commonly used materials in both old and modern times, the physical understanding of the formation and stability of iron and iron carbide phases is still in a premature state [1-5].

In the early 1950s, Hofer and Cohn investigated the formation of iron carbide precipitates in quenched steels $[1,2]$. They observed that the first stage of carbide formation occurs at temperatures between 370 and $470 \mathrm{~K}$, where $\eta$ - $\mathrm{Fe}_{2} \mathrm{C}$ forms first. The second stage occurs at around $550 \mathrm{~K}$, where the retained austenite decomposes into ferrite and $\theta-\mathrm{Fe}_{3} \mathrm{C}$. In the third stage (470 to $720 \mathrm{~K}$ ), $\theta-\mathrm{Fe}_{3} \mathrm{C}$ and $\chi-\mathrm{Fe}_{5} \mathrm{C}_{2}$ phases precipitate. Above $720 \mathrm{~K}$ only $\theta-\mathrm{Fe}_{3} \mathrm{C}$, cementite, is formed. Slowly cooling steels contain mainly ferrite and cementite [2]. $\theta-\mathrm{Fe}_{3} \mathrm{C}$ was also found during carburization of iron [6]. Recent work shows that $\theta-\mathrm{Fe}_{3} \mathrm{C}$ may play an important role in Earth's core as well [7]. It was shown that cementite, as a weak magnet, shows almost no thermal expansion (Invar behavior) at the temperature below its Curie temperature [8,9]. Notwithstanding many theoretical efforts, very basic questions on steel remain unanswered $[3-5,10]$. One prominent question is why the cementite phase is observed much more frequently than other carbide phases in steel.

Iron carbides exhibit a rich variety of crystal structures and physical properties. Up to now, about a dozen structural models for binary iron carbides have been obtained or proposed by experimentalists, covering a wide variety of crystal structures [2,11-14].

To explain the predominance of cementite in steel, theoretical approaches, especially the electronic densityfunctional theory (DFT) techniques, can provide essential insights. Here we present a systematic DFT study on the formation and stability of binary iron carbides within the generalized gradient approximation, with details in Refs. $[15,16]$. Various structural models and magnetic orderings
PACS numbers: 61.50.Lt, 64.60.My, 71.15.Nc, 75.50.Bb

are examined for, in total, 17 carbide phases, and the factors that are considered to be important for the stability are discussed. Finally, we address the changes in the relative stability caused by lattice vibrations and magnetic ordering at elevated temperatures, which turn out to be of major importance to the formation of carbides.

We first performed total energy calculations for the elemental solids $\alpha$-Fe and diamond. $\alpha$-Fe has a bec structure $[15,16]$. The calculations give a lattice parameter $a=$ $2.831 \AA$ and local moment $M=2.21 \mu_{B} / \mathrm{Fe}$ which agree well with the experimental values $(a=2.866 \AA$ and $M=$ $2.12 \mu_{B} /$ atom). We performed calculations for diamond and added a correction term $(-17 \mathrm{meV} / \mathrm{C})$ in order to obtain the enthalpy for graphite [16] The calculated diamond lattice parameter is $3.5713 \AA$, which agrees with the experimental value $(3.5668 \AA$ at $300 \mathrm{~K})$.

Taking the calculated energies of the elemental solids, we can obtain the formation enthalpy $\left(\Delta H_{f}\right)$ of $\mathrm{Fe}_{n} \mathrm{C}_{m}$ at $T=0 \mathrm{~K}$ and $P=0 \mathrm{~Pa}$, using the following formula whereby the zero-point vibration contribution is ignored:

$$
\Delta H_{f}=\left\{E\left(\mathrm{Fe}_{n} \mathrm{C}_{m}\right)-[n E(\mathrm{Fe})+m E(\mathrm{C})]\right\} /(n+m) .
$$

Figure 1 shows the calculated formation enthalpies for the iron carbides with $\Delta H_{f}<150 \mathrm{meV} /$ atom. The formation enthalpies for all the iron carbides are positive. This indicates that at $T=0 \mathrm{~K}$ and $P=0 \mathrm{~Pa}$, none of the iron carbides are stable relative to the elemental solids (graphite and $\alpha-\mathrm{Fe}$ ), in agreement with experiments [2].

As shown in Fig. 1, the formation enthalpies of $\eta-\mathrm{Fe}_{2} \mathrm{C}$ $(17.3 \mathrm{meV} /$ atom $), \quad \chi-\mathrm{Fe}_{5} \mathrm{C}_{2} \quad(18.6 \mathrm{meV} /$ atom $), \quad$ and $\theta-\mathrm{Fe}_{3} \mathrm{C}(20.6 \mathrm{meV} /$ atom $)$ are among the lowest. All three compounds have similar hexagonal close-packed (hcptype) $\mathrm{Fe}$ sublattices [14]. The calculations also show that the orthorhombic $\eta-\mathrm{Fe}_{2} \mathrm{C}$ phase is the most stable carbide, while the hexagonal form $(\varepsilon-) \mathrm{Fe}_{2} \mathrm{C}$ within a simple hcp 


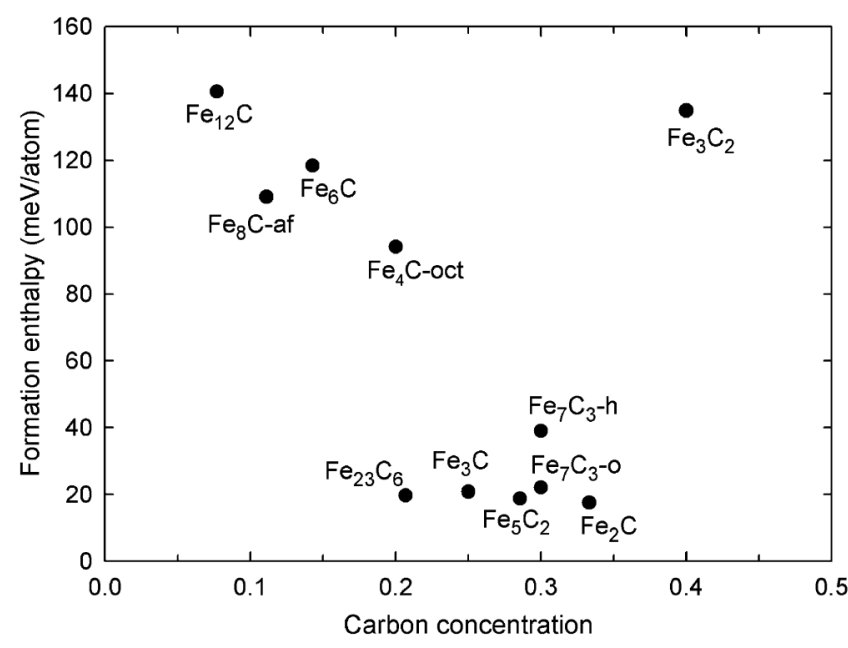

FIG. 1. Formation enthalpies, $\Delta H_{f}$ [see Eq. (1)], for iron carbides $\left(\mathrm{Fe}_{n} \mathrm{C}_{m}\right)$ with respect to the elemental solids (graphite and $\alpha$-Fe) from first-principles calculations. For clarity, the data for hexagonal $\varepsilon-\mathrm{Fe}_{x} C(x=2,3)$ are not included.

lattice as proposed by Jack in 1948 has a much higher formation enthalpy ( $\sim 120 \mathrm{meV} /$ atom) [17]. A hexagonal lattice $\varepsilon-\mathrm{Fe}_{3} \mathrm{C}$ was calculated to be slightly less stable than $\theta-\mathrm{Fe}_{3} \mathrm{C} \quad\left(\Delta H_{f}=25.1 \mathrm{meV} /\right.$ atom vs $20.6 \mathrm{meV} /$ atom $)$ $[1,2,11]$. Another relatively stable carbide is $\mathrm{Fe}_{23} \mathrm{C}_{6}$, which has a formation enthalpy comparable to that of $\theta-\mathrm{Fe}_{3} \mathrm{C}$ (19.5 meV/atom vs $20.6 \mathrm{meV} /$ atom). This carbide does not have a hcp-type Fe sublattice and has been discussed elsewhere [16].

As shown in Fig. 1, the calculations also show a trend of stability, $-\Delta H_{f}$, from high to low: $-\Delta H_{f}\left(\eta-\mathrm{Fe}_{2} \mathrm{C}\right)>$ $-\Delta H_{f}\left(\chi-\mathrm{Fe}_{5} \mathrm{C}_{2}\right)>-\Delta H_{f}\left(\theta-\mathrm{Fe}_{3} \mathrm{C}\right)$. However, experimentally cementite is formed much more frequently in carbon steels than $\eta-\mathrm{Fe}_{2} \mathrm{C}$ or/and $\chi-\mathrm{Fe}_{5} \mathrm{C}_{2}$.

Conventionally, one of the most important factors stabilizing the $\theta-\mathrm{Fe}_{3} \mathrm{C}$ phase in steels is considered to be the interfacial energy between $\theta-\mathrm{Fe}_{3} \mathrm{C}$ and ferrite [2]. The interfacial energies are influenced, not only by the lattice matching as discussed by many experimentalists, but also by the chemical bonding at the interfaces. $\mathrm{Fe}_{23} \mathrm{C}_{6}$ has a small lattice mismatch with austenite [16], but is rarely observed in unalloyed steels. Analysis suggests that the high carbon solubility in austenite, the Fe vacancies needed for nucleation, and the distortion of the Fe planes at the metal-carbide interface are the culprits.

A careful analysis of the lattice matches between the iron carbides of the hcp family to the ferrite matrix has revealed that these carbides all have similar mismatches because their crystal structures are so similar. Hence it is unlikely that the lattice mismatch can explain the prevalence of cementite relative to $\eta-\mathrm{Fe}_{2} \mathrm{C}$ and $\chi-\mathrm{Fe}_{5} \mathrm{C}_{2}$.

Magnetism plays an important role in $\mathrm{Fe}$ and the iron carbides. Our calculations show that most of the iron carbides, including all the members of the hcp family, exhibit ferromagnetism, in spite of the fact that the ground state of a pure hcp-Fe crystal has a spiral magnetic ordering with a small local moment [18]. Below we discuss the influence of magnetism and lattice vibration on the stability of iron carbides of the hep family.

Figure 1 shows that two iron carbides have lower formation enthalpies than that of cementite: $\eta-\mathrm{Fe}_{2} \mathrm{C}$ and $\chi-\mathrm{Fe}_{5} \mathrm{C}_{2} . \eta-\mathrm{Fe}_{2} \mathrm{C}$ is the most observed iron carbide at low temperature, while $\chi-\mathrm{Fe}_{5} \mathrm{C}_{2}$ is less commonly observed in steel, and its Curie temperature is not known. Therefore, we only compare the temperature-dependent relative stability of $\eta-\mathrm{Fe}_{2} \mathrm{C}$ and $\theta-\mathrm{Fe}_{3} \mathrm{C}$. Hofer and Cohen [1] reported that cementite is formed from $\eta-\mathrm{Fe}_{2} \mathrm{C}$ and $\alpha$-Fe:

$$
\eta-\mathrm{Fe}_{2} \mathrm{C}+\alpha-\mathrm{Fe} \leftrightarrow \theta-\mathrm{Fe}_{3} \mathrm{C} .
$$

In order to determine the relative stability of $\theta-\mathrm{Fe}_{3} \mathrm{C}$ with respect to $\eta-\mathrm{Fe}_{2} \mathrm{C}$ and $\alpha$-Fe, we have calculated both the magnetic contribution and the lattice vibration contribution (including zero-point vibrations) to the stability of these three phases. The Gibbs energy can be separated into two contributions [19], as

$$
G_{\text {total }}(T)=G_{\text {nonmagn }}(T)+G_{\text {magn }}(T) .
$$

The nonmagnetic terms include contributions from lattice vibrations and electronic excitations. The lattice vi-

TABLE I. Calculated magnetic moments $\left(S_{a}, \mu_{B} / \mathrm{Fe}\right)$ and calculated valence energy $E_{\text {cal }}$ [eV per formula unit (f.u.]) and zero-point energy (ZPE, meV/atom) at zero $K$. Experimental Curie temperatures $\left(T_{c}\right)$ for iron and the iron carbides. The magnetic entropy according to $S_{\mathrm{mag}}=R \ln \left(1+S_{a}\right)$ [20]. $p, f_{s}, k_{f}, k_{p}$ are parameters for magnetic specific heat [Eqs. (3) and (4), see text for details].

\begin{tabular}{lccccc}
\hline \hline Phase & $\alpha$-Fe & $\theta-\mathrm{Fe}_{3} \mathrm{C}$ & $0-\mathrm{Fe}_{7} \mathrm{C}_{3}$ & $\chi-\mathrm{Fe}_{5} \mathrm{C}_{2}$ & $\eta-\mathrm{Fe}_{2} \mathrm{C}$ \\
\hline$E_{\text {cal }}(\mathrm{eV} /$ f.u. $)$ & -8.310 & -33.960 & -85.288 & -59.645 & -25.681 \\
$\mathrm{ZPE}(\mathrm{meV} /$ atom $)$ & 41.9 & 53.3 & $\ldots$ & $\cdots$ & 64.0 \\
$S_{a}\left(\mu_{B} / \mathrm{Fe}\right)$ & 2.21 & 1.86 & 1.72 & 1.69 & 1.60 \\
$S_{\mathrm{mag}}(R / \mathrm{Fe})$ & 1.166 & 1.051 & 1.001 & 0.990 & 0.956 \\
$T_{c}(\mathrm{~K})^{\mathrm{ref}}$ & 1041 & $483[8,21]$ & $520[21]$ & $\cdots$ & $540[22]$ \\
$p^{20}$ & 1 & 2 & 2 & 2 & 2 \\
$f_{s}^{20}$ & 0.105 & 0.105 & 0.105 & 0.105 & 0.105 \\
$k_{f}[\mathrm{~J} /(\mathrm{mol} \mathrm{K}) / \mathrm{Fe}]$ & 35.36 & 33.72 & 30.35 & $\cdots$ & 28.98 \\
$k_{p}[\mathrm{~J} /(\mathrm{mol} \mathrm{K}) / \mathrm{Fe}]$ & 8.15 & 15.53 & 13.98 & $\cdots$ & 13.35 \\
\hline \hline
\end{tabular}


bration term can be separated in specific heat at constant volume $\left(C_{v}\right)$ and thermal expansion effects $\left(C_{p}-C_{v}\right)$.

A measure for the relative stability of an iron carbide (represented by $i$ ) to the $\theta-\mathrm{Fe}_{3} \mathrm{C}$, cementite (noted by $c$ ), is

$$
\begin{aligned}
\Delta G_{\text {total }}^{i-c}(T)= & \Delta G_{\text {total }}^{i-c}(0)+\int\left(C_{p}{ }^{i}-C_{p}{ }^{c}\right) d T \\
& -T \int\left(C_{p}{ }^{i}-C_{p}{ }^{c}\right) / T d T .
\end{aligned}
$$

Here, $\Delta G_{\text {total }}^{i-c}(0)$ is the difference of free energies, being equal to the difference of formation enthalpies $\left(\Delta H^{i-c}\right)$ at $T=0 \mathrm{~K}$. Because of the structural similarity of the members of the hcp family, no significant differences are to be expected in the electronic and thermal expansion terms to the Gibbs energy. Therefore, the only really significant difference in the Gibbs energy of the various iron carbides originates from lattice vibrations and magnetism of the $\mathrm{Fe}$ sublattices.

Umemoto and colleagues measured the specific heat cementite [8]. A peak positioned at the Curie temperature (about $483 \mathrm{~K}$ ) is observed, in agreement with the CurieWeiss behavior of this magnetic material.

Table I lists the calculated magnetic moments of iron atoms in the carbides, at $T=0 \mathrm{~K}$ and $P=0 \mathrm{~Pa}$. With decreasing carbon concentration, the magnetic moment $\left(S_{a}\right)$ per $\mathrm{Fe}$ atom increases moderately, and $\theta-\mathrm{Fe}_{3} \mathrm{C}$ has the highest value.

Chuang and coworkers studied the contribution of the Curie-Weiss magnetic behavior to specific heat for ferrite and austenite [23]. The Curie-Weiss specific heat $\left(C_{m}\right)$, below and above the Curie temperature $T_{c}$, is commonly expressed as [23]

$$
\begin{aligned}
C_{m}=k_{f}\left(T / T_{c}\right) \exp \left[-4\left(1-T / T_{c}\right)\right] & \left(T<T_{c}\right),(5 \mathrm{a}) \\
C_{m}=k_{p}\left(T / T_{c}\right) \exp \left[8 p\left(1-T / T_{c}\right)\right] & \left(T>T_{c}\right) .(5 \mathrm{~b})
\end{aligned}
$$

The parameters $k_{f}$ and $k_{p}$ are determined by the Curie temperature $T_{c}$, the magnetic entropy $S_{\mathrm{mag}}$, and the fraction $\left(f_{s}\right)$ of magnetic entropy above the Curie temperature, with the formulas (i) $k_{f}=4\left(1-f_{s}\right) S_{\mathrm{mag}} /(1-\exp ([-4])$ and (ii) $k_{p}=8 p f_{s} S_{\mathrm{mag}}$, where $S_{\mathrm{mag}}=R \ln \left(1+S_{a}\right)$ [20,23] and $f_{s}=0.105$ [23], and $p=2$ is used as a parameter for the hcp structures, due to the strong similarity between the local structure of the Fe sublattices to that of the fcc lattice[23].

The phonon spectrum of $\alpha$-Fe is well established both experimentally and theoretically [24-26]. The thermodynamic properties of cementite including the magnetic contribution were studied as well [27]. For the sake of systematic study, we performed phonon calculations for $\alpha$-Fe, $\eta-\mathrm{Fe}_{2} \mathrm{C}$, and $\theta-\mathrm{Fe}_{3} \mathrm{C}$ employing the harmonic approximation [28] with the force constants obtained using the DFT-generalized-gradient-approximation method. The calculated results of the phonon spectra and phonon density of states are very close to those in the literature for
$\alpha$-Fe [24,25] and $\theta-\mathrm{Fe}_{3} \mathrm{C}$ [26]. The calculated phonon spectrum of $\eta-\mathrm{Fe}_{2} \mathrm{C}$, being similar to that of $\theta-\mathrm{Fe}_{3} \mathrm{C}$, consists of three separated parts: the low energy part $(0$ to $40 \mathrm{meV}$ ) from $\mathrm{Fe}$ contribution, and two rather narrow bands at high energies of $\mathrm{C}$ contributions. Details of the

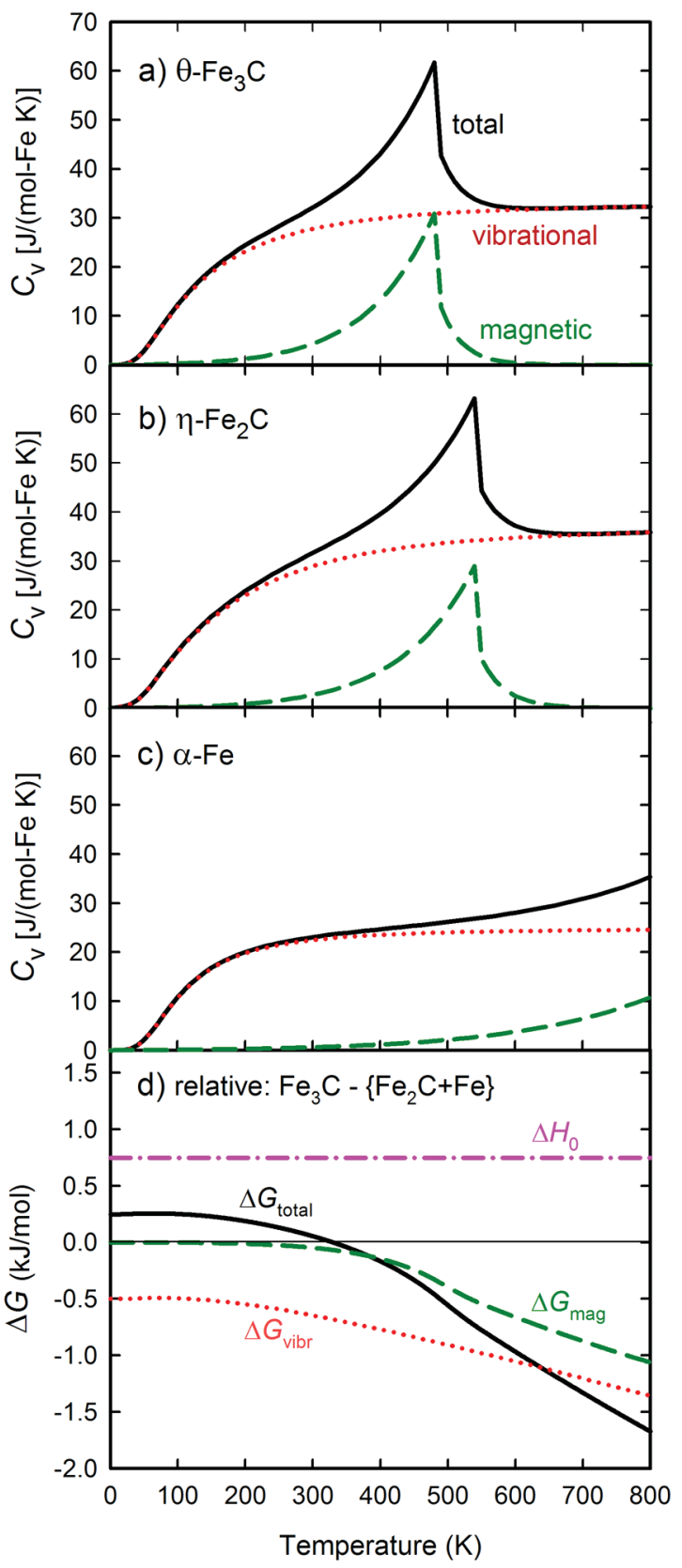

FIG. 2 (color). Specific heat $\mathrm{C}_{v}$ of $\theta-\mathrm{Fe}_{3} \mathrm{C}(\mathrm{a}), \eta-\mathrm{Fe}_{2} \mathrm{C}(\mathrm{b})$, and $\alpha$-Fe (c) obtained from the phonon calculations (red dotted line) and from Curie-Weiss magnetism as given by Eq. (5) (green dashed line); the sum of both terms is shown by the black solid line. Figure (d) shows the free energy difference $\Delta G$ between $\mathrm{Fe}_{3} \mathrm{C}$ and $\left\{\mathrm{Fe}_{2} \mathrm{C}+\mathrm{Fe}\right\}$. The contribution from the Curie-Weiss magnetism (lattice vibrations) is shown by the green dashed (red dotted) line, while the formation energy difference is given by the magenta dash-dotted line. The total free energy difference between $\mathrm{Fe}_{3} \mathrm{C}$ and $\left\{\mathrm{Fe}_{2} \mathrm{C}+\mathrm{Fe}\right\}$ is shown by the black solid line. 
calculations and phonon spectra of the phases will be discussed elsewhere.

Figures 2(a)-2(c) show the computed specific heat of $\theta-\mathrm{Fe}_{3} \mathrm{C}, \eta-\mathrm{Fe}_{2} \mathrm{C}$, and $\alpha$-Fe, respectively. The magnetic contribution to the specific heat was obtained using Eq. (5) with the parameters in Table I. The free energy of an iron carbide is obtained from the computed specific heat.

The free energy difference $\left[\Delta G^{i-c}(T)_{\text {total }}\right]$ between $\mathrm{Fe}_{3} \mathrm{C}$ and $\left\{\eta-\mathrm{Fe}_{2} \mathrm{C}+\mathrm{Fe}\right\}$ can now be evaluated by substituting all values into Eqs. (3) and (4). The result is displayed in Fig. 2(d). The vibrational contribution to $\Delta G^{i-c}(T)_{\text {total }}$ is $-0.50 \mathrm{~kJ} / \mathrm{mol}$ at $T=0 \mathrm{~K}$ because of the zero-point vibration contributions, and then decreases gradually with increasing temperature. On the other hand, the contribution from magnetic ordering (Curie-Weiss behavior) is zero at $T=0 \mathrm{~K}$, remains nearly zero up to $300 \mathrm{~K}$, and then starts to decrease significantly. Considering the total energy difference [black solid curve in Fig. 2(d)], it is clear that $\eta$ - $\mathrm{Fe}_{2} \mathrm{C}$ is more stable at low temperatures. With increasing temperature, the energy difference between $\eta-\mathrm{Fe}_{2} \mathrm{C}$ and $\theta-\mathrm{Fe}_{3} \mathrm{C}$ becomes smaller, and above $330 \mathrm{~K}, \theta-\mathrm{Fe}_{3} \mathrm{C}$ is more stable than $\eta-\mathrm{Fe}_{2} \mathrm{C}$.

Figures 1 and 2(d), show that, at low temperature, nucleation and crystal growth of iron carbide precipitates will occur with initial formation of $\eta-\mathrm{Fe}_{2} \mathrm{C}$, due to its low formation enthalpy. During heating, contributions from lattice vibrations and magnetism increase the stability of $\theta-\mathrm{Fe}_{3} \mathrm{C}$. When the temperature is high enough to overcome the transformation barrier, $\eta-\mathrm{Fe}_{2} \mathrm{C}$ transforms to $\theta-\mathrm{Fe}_{3} \mathrm{C}$. On the other hand, for slow-cooling processes at elevated temperatures $\theta-\mathrm{Fe}_{3} \mathrm{C}$ is formed without $\eta-\mathrm{Fe}_{2} \mathrm{C}$ as an intermediary. Recently, Schneider and Inden found that the activation energy barrier for carbon diffusion in cementite is about $1.79 \mathrm{eV} /$ atom [29] The activation energy barriers will prevent transformation to other phases, such as $\eta-\mathrm{Fe}_{2} \mathrm{C}$ and/or $\chi-\mathrm{Fe}_{5} \mathrm{C}_{2}$ at low temperature. This clarifies the experimental observation of preferential $\theta-\mathrm{Fe}_{3} \mathrm{C}$ formation during steel fabrication processes.

Furthermore, it can be concluded that in comparison with other materials, the stability of iron carbides is rather subtle and that magnetism can have a determining influence. Therefore, a careful assessment of temperaturedependent energetics is required for making reliable stability predictions.

The research was carried out under the Projects No. MC5.06280 and No. MC.05239 in the framework of the Materials Innovation Institute M2i (www.m2i.nl), the Netherlands.

*Corresponding author: Tel./fax:+31 15 2781536/+31 15 2786600.

cfang@tudelft.nl
[1] L. J. E. Hofer and E. M. Cohn, Nature (London) 167, 977 (1951).

[2] J. W. Christian, The Theory of Transformations in Metals and Alloys (Pergamon Press, Amsterdam, 2002).

[3] T.E. Jones, M.E. Eberhart, and D. P. Clougherty, Phys. Rev. Lett. 100, 017208 (2008).

[4] D. W. Boukhvalov, Yu. N. Gornostyrev, M. I. Katsnelson, and A.I. Lichtenstein, Phys. Rev. Lett. 99, 247205 (2007).

[5] J. W. Morris, Jr., Science 320, 1022 (2008).

[6] M. Audier, P. Bowen, and W. Jones, J. Cryst. Growth 64, 291 (1983).

[7] B. J. Wood, Earth Planet Sci. Lett. 117, 593 (1993).

[8] M. Umemoto, Z. G. Liu, K. Matsuyama, and K. Tsuchiya, Scr. Mater. 45, 391 (2001).

[9] Y. Kimura, T. Inoue, F. Yin, and K. Tsuzaki, Science 320, 1057 (2008).

[10] L. Vitos, P. A. Korzhavyi, and B. Johansson, Nature Mater. 2, 25 (2003).

[11] H. J. Goldschmidt, J. Iron Steel Inst. A 160, 345 (1948).

[12] S. Nagakura and S. Oketani, Trans. Iron Steel Inst. Jpn. 8, 265 (1968).

[13] E. R. D. Scott, Nature (London) 229, 61 (1971).

[14] B. G. Hyde, S. Andersson, M. Bakker, C. M. Plug, and M. O'Keeffe, Prog. Solid State Chem. 12, 273 (1979).

[15] All calculations were carried out using the VASP code employing the density-functional theory within the projector-augmented wave method. The generalized gradient approximation by J.P. Perdew, K. Burke, and M. Ernzerhof, Phys. Rev. Lett. 77, 3865 (1996) was employed for the exchange and correlation energy terms. The cutoff energy of the wave functions was $500 \mathrm{eV}$. Dense $k$ meshes were used, e.g., a $12 \times 12 \times 12$ grid with $84 k$ points in the irreducible Brillouin zone of $\theta-\mathrm{Fe}_{3} \mathrm{C}$ using the $\mathrm{H}$. J. Monkhorst and J. D. Pack method, Phys. Rev. B 13, 5188 (1976). Details of settings are described in Ref. [16].

[16] C. M. Fang, M. A. van Huis, M. H. F. Sluiter, and H. W. Zandbergen, Acta Mater. 58, 2968 (2010).

[17] K. H. Jack, Proc. R. Soc. A 195, 56 (1948).

[18] M. Friák and M. Šob, Phys. Rev. B 77, 174117 (2008).

[19] C. Zener, J. Appl. Phys. 22, 372 (1951).

[20] J. A. Hofmann, A. Paskin, K. J. Tauer, and R. Weiss, J. Phys. Chem. Solids 1, 45 (1956).

[21] A. Tsuzuki, S. Sago, S. Hirano, and S. Naka, J. Mater. Sci. 19, 2513 (1984).

[22] E. M. Cohn and L. J. E. Hofer, J. Am. Chem. Soc. 72, 4662 (1950).

[23] Y. Y. Chuang, R. Schmid, and Y. A. Chang, J. Mater. Sci. 16, 153 (1985).

[24] V. J. Minkiewicz, G. Shirane, and R. Nathans, Phys. Rev. 162, 528 (1967).

[25] H. K. Mao et al., Science, 292, 914 (2001).

[26] C. Jiang, S. G. Srinivasan, A. Caro, and S. A. Maloy, J. Appl. Phys. 103, 043502 (2008).

[27] B. Hallstedt et al., CALPHAD: Comput. Coupling Phase Diagrams Thermochem. 34, 129 (2010).

[28] D. Alfè, Comput. Phys. Commun. 180, 2622 (2009).

[29] A. Schneider and G. Inden, CALPHAD: Comput. Coupling Phase Diagrams Thermochem. 31, 141 (2007). 